\title{
Construction of Bivariate Nonseparable Compactly Supported Orthogonal Wavelets
}

\author{
Jinsong Leng, ${ }^{1}$ Tingzhu Huang, ${ }^{1}$ and Carlo Cattani ${ }^{2}$ \\ ${ }^{1}$ School of Mathematical Sciences, University of Electronic Science and Technology of China, Chengdu 610054, China \\ ${ }^{2}$ Department of Mathematics, University of Salerno, Via Ponte Don Melillo, 84084 Fisciano, Italy \\ Correspondence should be addressed to Jinsong Leng; jslengjs@gmail.com
}

Received 7 January 2013; Accepted 24 January 2013

Academic Editor: Sheng-Yong Chen

Copyright (c) 2013 Jinsong Leng et al. This is an open access article distributed under the Creative Commons Attribution License, which permits unrestricted use, distribution, and reproduction in any medium, provided the original work is properly cited.

\begin{abstract}
A method for constructing bivariate nonseparable compactly supported orthogonal scaling functions, and the corresponding wavelets, using the dilation matrix $M:=2^{n} \square=2^{n}\left[\begin{array}{ll}1 & 0 \\ 0 & 1\end{array}\right],\left(d=\operatorname{det} M=2^{2 n} \geq 4, n \in \mathbb{N}\right)$ is given. The accuracy and smoothness of the scaling functions are studied, thus showing that they have the same accuracy order as the univariate Daubechies low-pass filter $m_{0}(\omega)$, to be used in our method. There follows that the wavelets can be made arbitrarily smooth by properly choosing the accuracy parameter $r$.
\end{abstract}

\section{Introduction}

Wavelet analysis (see $[1,2])$ has become a powerful tool in neural networks, harmonic analysis, numerical analysis, and signal processing, especially in the area of image compression [3]. The wavelet transform is a simple and practical mathematical tool that cuts up data or functions into different frequency components and then studies each component with a resolution matched to its scale. The main feature of the wavelet transform is to hierarchically decompose general functions, as a signal or a process, into a set of approximation functions with different scales $[1,2,4,5]$.

Wavelet theory is closely related to subband coding, and it provides a functional space structure for subband coding, often leading to a better understanding of signals.

Univariate wavelets have found successful applications in signal processing [5-7]. However, in order to apply wavelet methods to data processing and to analyze multidimensional signals, such as image processing, we have to construct multivariate wavelets. The most commonly used method to define multivariate wavelets is the tensor product of univariate wavelets $[4,8-10]$. This construction leads to a separable wavelet function having, as a major drawback, the horizontal and vertical (obliged) directions, while usually in imaging process the information contents spread along all directions, in a nonisotropic way. Much efforts have been spent for constructing multivariate nonseparable compactly supported orthogonal wavelets (see, e.g., [11-26]); however, the generalization to higher dimensions is not a simple task, so that a general simple methodology is still missing. However, it is possible to characterize the construction of bivariate scaling functions by the two scale difference equation, which in 1 dimension is

$$
\varphi(x)=\sum_{k \in \mathbb{Z}} c_{k} \varphi(2 x-k), \quad(x \in \mathbb{R})
$$

and in 2 dimensions

$$
\varphi(\mathbf{x})=\sum_{\mathbf{k} \in \mathbb{Z}^{2}} \varphi(M \mathbf{x}-\mathbf{k}), \quad\left(\mathbf{x} \in \mathbb{R}^{2}\right)
$$

depends on the coefficients of a suitable second-order dilation matrix $M$. Usually $[9,11-13]$ the determinant of this matrix is 2. For example, Ayache [11, 12] proposed two methods for constructing bivariate nonseparable compactly supported orthonormal wavelets. The wavelets constructed using one of the methods are called semiseparable wavelets. Belogay and Wang [13] constructed bivariate nonseparable compactly supported orthonormal wavelets using the dilation matrix $M=\left[\begin{array}{ll}0 & 2 \\ 1 & 0\end{array}\right]$. Belogay and Wang gave the sufficient condition for the low-pass filters constructed by their method to generate nonseparable compactly supported orthogonal 
accurate scaling function with arbitrarily high smoothness. However, for proving the accuracy these authors used the matrix $M^{2}= \pm 2 \rrbracket$. Moreover, their method has been modified by Lai [18] to construct nonseparable wavelets using the dilation matrix $\left[\begin{array}{ll}2 & 0 \\ 0 & 2\end{array}\right]$ with determinant equal to 4 . He and Lai [16] constructed many examples of nonseparable orthogonal wavelets. Lai and Roach [19] provided a method to construct bivariate nonseparable compactly supported wavelets by using the method of symmetry. Stanhill and Zeevi $[24,25]$ provided other methods for constructing bivariate nonseparable orthogonal wavelets.

By combining the Belogay and Wang method, the Lai method, and the Ayache method, we present another method for constructing bivariate nonseparable orthogonal wavelets using the dilation matrix $M=2 \rrbracket=\left[\begin{array}{ll}2 & 0 \\ 0 & 2\end{array}\right]$. Our method holds true also for dilation matrix of the form

$$
M=2^{n} \square=2^{n}\left[\begin{array}{ll}
1 & 0 \\
0 & 1
\end{array}\right] \quad(n \in \mathbb{N})
$$

being $\square:=\left[\begin{array}{ll}1 & 0 \\ 0 & 1\end{array}\right]$ the identity matrix, so that

$$
d=\operatorname{det} M=2^{2 n} \quad(n \in \mathbb{N}) .
$$

It follows that the two-scale difference equation simplifies into

$$
\varphi(\mathbf{x})=\sum_{\mathbf{k} \in \mathbb{Z}^{2}} \varphi\left(2^{n} \mathbf{x}-\mathbf{k}\right) \quad\left(\mathbf{x} \in \mathbb{R}^{2}, n \in \mathbb{N}\right)
$$

which is more alike the corresponding equation in 1 dimension. The minor drawback is that for the multivariate scaling function there exist $d-1$ corresponding wavelets (see, e.g., [9]), so that for a matrix with determinant 4 there exist, together with a scaling function, 3 wavelet functions (instead of 1 wavelet when $d=2$ ). However, due to the special form of the dilation matrix, we can take into account the results of [11-13] and show that the scaling function constructed by our method has the same accuracy as the univariate Daubechies low-pass filter to be used in our method. Following [13] we will also show that the smoothness of our bivariate scaling function can be manipulated by properly choosing the accuracy parameter $r$.

The paper is organized as follows. In Section 2, we introduce some notations and briefly recall some basic elements of the theory of bivariate multiresolution analysis and bivariate orthogonal wavelets. In Section 3, we provide a method for constructing a class of bivariate nonseparable compactly supported orthogonal filter banks. Then we study the accuracy and the smoothness of the scaling function constructed by our method. Finally, we give an example of the bivariate nonseparable compactly supported scaling function.

\section{Preliminaries}

Let $\mathbb{Z}$ and $\mathbb{R}$ be the sets of all integers and real numbers, respectively. Denote

$$
\begin{gathered}
L^{2}\left(\mathbb{R}^{2}\right):=\left\{f(\mathbf{x}): \int_{\mathbb{R}^{2}}|f(\mathbf{x})|^{2} d \mathbf{x}<\infty, \mathbf{x} \in \mathbb{R}^{2}\right\}, \\
\ell^{2}\left(\mathbb{Z}^{2}\right)=\left\{\left\{C_{\mathbf{n}}\right\}_{\mathbf{n} \in \mathbb{Z}^{2}}: \sum_{\mathbf{n} \in \mathbb{Z}^{2}}\left|C_{\mathbf{n}}\right|^{2}<\infty\right\} .
\end{gathered}
$$

The Fourier transform of $f(\mathbf{x})$ is defined by

$$
\widehat{f}(\boldsymbol{\omega})=\int_{\mathbb{R}^{2}} f(\mathbf{x}) e^{-i \boldsymbol{\omega} \cdot \mathbf{x}} d \mathbf{x},
$$

where $\boldsymbol{\omega}, \mathbf{x} \in \mathbb{R}^{2}, i=\sqrt{-1}$, and $\boldsymbol{\omega} \cdot \mathbf{x}:=\omega_{1} x_{1}+\omega_{2} x_{2}$ is the dot product. by

For $f(\mathbf{x}), g(\mathbf{x}) \in L^{2}\left(\mathbb{R}^{2}\right)$, their inner product is defined

$$
\langle f(\mathbf{x}), g(\mathbf{x})\rangle:=\int_{\mathbb{R}^{2}} f(\mathbf{x}) g(\mathbf{x}) d \mathbf{x},
$$

which is explicitly

$$
\left\langle f\left(x_{1}, x_{2}\right), g\left(x_{1}, x_{2}\right)\right\rangle:=\int_{\mathbb{R}^{2}} f\left(x_{1}, x_{2}\right) g\left(x_{1}, x_{2}\right) d x_{1} d x_{2} .
$$

A function $f(\mathbf{x}) \in L^{2}\left(\mathbb{R}^{2}\right)$ is called orthogonal if the set of its translate $\{f(\mathbf{x}-\mathbf{k})\}_{\mathbf{k} \in \mathbb{Z}^{2}}$ is orthogonal; that is,

$$
\langle f(\mathbf{x}-\mathbf{k}), f(\mathbf{x}-\mathbf{h})\rangle=\delta_{\mathbf{k h}},
$$

where $\mathbf{k}, \mathbf{h} \in \mathbb{Z}^{2}$ and $\delta_{\mathbf{k h}}$ is the Kronecker notation defined by $\delta_{\mathbf{k h}}=1$ if $\mathbf{k}=\mathbf{h}$ and $\delta_{\mathbf{k h}}=0$ if $\mathbf{k} \neq \mathbf{h}$, which is explicitly

$$
\left\langle f\left(x_{1}-k_{1}, x_{2}-k_{2}\right), f\left(x_{1}-h_{1}, x_{2}-h_{2}\right)\right\rangle=\delta_{k_{1} h_{1}} \delta_{k_{2} h_{2}} .
$$

The whole construction of wavelets is based on the multiresolution analysis (MRA) and its corresponding axioms, which can be easily extended to the bivariate multiresolution analysis as follows. Let $\varphi(\mathbf{x}) \in L^{2}\left(\mathbb{R}^{2}\right)$ satisfy the following refinement equation:

$$
\varphi(\mathbf{x})=\sum_{\mathbf{k} \in \mathbb{Z}^{2}} p_{\mathbf{k}} \varphi(2 \mathbf{x}-\mathbf{k})
$$

where $\left\{p_{\mathbf{k}}\right\}_{\mathbf{k} \in \mathbb{Z}^{2}}$ is a real constant sequence.

We assume that $p_{\mathbf{k}} \neq 0$ for only finitely many $\mathbf{k} \in \mathbb{Z}^{2}$ (ensuring that $\varphi(\mathbf{x})$ has compact support).

Define a closed subspace $V_{j} \subset L^{2}\left(\mathbb{R}^{2}\right)$ by

$$
V_{j}=\operatorname{clos}_{L^{2}\left(\mathbb{R}^{2}\right)}\left(\operatorname{span}\left\{2^{j} \varphi\left(2^{j} \mathbf{x}-\mathbf{k}\right): \mathbf{k} \in \mathbb{Z}^{2}\right\}\right), \quad j \in \mathbb{Z} .
$$

Definition 1. One says that $\varphi(\mathbf{x})$ in (12) generates a bivariate multiresolution analysis (MRA) $\left\{V_{j}\right\}_{j \in \mathbb{Z}}$ of $L^{2}\left(\mathbb{R}^{2}\right)$ if the sequence $\left\{V_{j}\right\}_{j \in \mathbb{Z}}$ defined by (13) satisfies

(i) $\cdots \subset V_{-1} \subset V_{0} \subset V_{1} \cdots$;

(ii) $\bigcup_{j \in \mathbb{Z}} V_{j}$ is dense in $L^{2}\left(\mathbb{R}^{2}\right)$ and $\bigcap_{j \in \mathbb{Z}} V_{j}=\{0\}$, where 0 is the zero function;

(iii) $f(\mathbf{x}) \in V_{j} \Leftrightarrow f(2 \mathbf{x}) \in V_{j+1}, j \in \mathbb{Z}$;

(iv) $\{\varphi(\mathbf{x}-\mathbf{k})\}_{\mathbf{k} \in \mathbb{Z}^{2}}$ is an orthogonal basis of $V_{0}$.

The function $\varphi(\mathbf{x})$ is called an orthogonal scaling function of the bivariate multiresolution analysis $\left\{V_{j}\right\}_{j \in \mathbb{Z}}$. 

have

By taking the Fourier transform for both sides of (12), we

$$
\widehat{\varphi}(\xi, \eta)=P_{0}\left(z_{1}, z_{2}\right) \widehat{\varphi}\left(\frac{\xi}{2}, \frac{\eta}{2}\right), \quad \xi, \eta \in \mathbb{R}
$$

where

$$
P_{0}\left(z_{1}, z_{2}\right)=\sum_{(k, l) \in \mathbb{Z}^{2}} p_{k, l} z_{1}^{k} z_{2}^{l}, \quad z_{1}:=e^{i \xi / 2}, z_{2}:=e^{i \eta / 2}
$$

is a Laurent polynomial satisfying $P_{0}(1,1)=1$.

The orthogonality condition (10) implies that

$$
\sum_{k_{1}, k_{2} \in\{0,1\} \times\{0,1\}}\left|P_{0}\left((-1)^{k_{1}} z_{1},(-1)^{k_{2}} z_{2}\right)\right|^{2}=1 \text {. }
$$

The function $P_{0}\left(z_{1}, z_{2}\right)$ is called a low-pass filter (refinement mask). By $(14)$ and $P_{0}(1,1)=1$, we get

$$
\widehat{\varphi}(\xi, \eta)=\prod_{k=1}^{\infty} P_{0}\left(e^{i \xi / 2^{k}}, e^{i \eta / 2^{k}}\right),
$$

so that the scaling function can be defined by a suitable choice of the low-pass filter.

Let $C(\xi, \eta)=P_{0}\left(z_{1}, z_{2}\right)$; the filter $P_{0}\left(z_{1}, z_{2}\right)$ satisfies Cohen's criterion [26] if there exists a compact fundamental domain $\Omega$ of the lattice $2 \pi \mathbb{Z}^{2}$ with the property

$$
C\left(\frac{\xi}{2^{j}}, \frac{\eta}{2^{j}}\right) \neq 0, \quad \forall j \geq 1, \forall(\xi, \eta) \in \Omega .
$$

Let us note that (16) is only a necessary condition for $\varphi(\mathbf{x})$ to be orthogonal. The condition (16) becomes also a sufficient condition if the filter $P_{0}\left(z_{1}, z_{2}\right)$ satisfies Cohen's criterion (18).

Let us now assume that there exist three closed subspaces $W_{0, i} \subset L^{2}\left(\mathbb{R}^{2}\right), i=1,2,3$, such that

$$
V_{1}=V_{0} \oplus W_{0,1} \oplus W_{0,2} \oplus W_{0,3}
$$

where $\oplus$ denotes orthogonal direct sum of spaces. We say that the compactly supported functions $\psi_{i}(\mathbf{x}), i=1,2,3$ are orthogonal wavelets if $\left\{\psi_{i}(\mathbf{x}-\mathbf{k})\right\}_{\mathbf{k} \in \mathbb{Z}^{2}}$ form orthogonal bases of $W_{0, i}, i=1,2,3$; that is,

$$
\begin{gathered}
W_{0, i}=\cos _{L^{2}\left(\mathbb{R}^{2}\right)}\left(\operatorname{span}\left\{\psi_{i}(\mathbf{x}-\mathbf{k}): \mathbf{k} \in \mathbb{Z}^{2}\right\}\right), \quad i=1,2,3, \\
\left\langle\psi_{i}(\mathbf{x}-\mathbf{k}), \psi_{j}(\mathbf{x}-\mathbf{l})\right\rangle=\delta_{i, j} \delta_{\mathbf{k}, l},
\end{gathered}
$$

where $i, j=1,2,3$ and $\mathbf{k}, l \in \mathbb{Z}^{2}$.

If there exist three Laurent polynomials $P_{i}\left(z_{1}, z_{2}\right), i=$ $1,2,3$, such that

$$
Q=\left[\begin{array}{llll}
P_{0}\left(z_{1}, z_{2}\right) & P_{0}\left(-z_{1}, z_{2}\right) & P_{0}\left(z_{1},-z_{2}\right) & P_{0}\left(-z_{1},-z_{2}\right) \\
P_{1}\left(z_{1}, z_{2}\right) & P_{1}\left(-z_{1}, z_{2}\right) & P_{1}\left(z_{1},-z_{2}\right) & P_{1}\left(-z_{1},-z_{2}\right) \\
P_{2}\left(z_{1}, z_{2}\right) & P_{2}\left(-z_{1}, z_{2}\right) & P_{2}\left(z_{1},-z_{2}\right) & P_{2}\left(-z_{1},-z_{2}\right) \\
P_{3}\left(z_{1}, z_{2}\right) & P_{3}\left(-z_{1}, z_{2}\right) & P_{3}\left(z_{1},-z_{2}\right) & P_{3}\left(-z_{1},-z_{2}\right)
\end{array}\right]
$$

is unitary, that is, $\operatorname{det} Q=1$, then the Fourier transforms of the functions $\psi_{i}(\mathbf{x}), i=1,2,3$, can be given by

$$
\widehat{\psi}_{i}(\xi, \eta)=P_{i}\left(e^{i \xi / 2}, e^{i \eta / 2}\right) \hat{\varphi}\left(\frac{\xi}{2}, \frac{\eta}{2}\right),
$$

and the three Laurent polynomials $P_{i}\left(z_{1}, z_{2}\right), i=1,2,3$, are called the high-pass filters (wavelet masks). The whole set of functions $\left\{P_{i}\left(z_{1}, z_{2}\right)\right\}_{0 \leq i \leq 3}$ is called a filter bank.

By (17) and (22), the construction of bivariate compactly supported orthogonal scaling function and the corresponding wavelets is reduced to the construction of filter bank $\left\{P_{i}\left(z_{1}, z_{2}\right)\right\}_{0 \leq i \leq 3}$ which satisfies (16), (18), and (21).

\section{Construction of Bivariate Nonseparable Orthogonal Wavelets}

3.1. Construction of the Filter Banks. According to Section 2, for constructing bivariate orthogonal scaling function and the corresponding wavelets, we mainly need to define the bivariate orthogonal filter bank $\left\{P_{i}\left(z_{1}, z_{2}\right)\right\}_{0 \leq i \leq 3}$ satisfying (16), (18), and (21).

Let us shortly recall the Belogay and Wang method, the Lai method, and the Ayache method for constructing bivariate nonseparable compactly supported orthogonal wavelets and their corresponding filter banks.

Belogay and Wang [13] constructed the bivariate nonseparable orthogonal scaling functions using the dilation matrix $\left[\begin{array}{ll}0 & 2 \\ 1 & 0\end{array}\right]$ with special coefficients: the coefficients are aligned along two adjacent rows. The corresponding low-pass filters have the form

$$
P_{0}\left(z_{1}, z_{2}\right)=A\left(z_{1}\right)+z_{2} B\left(z_{1}\right),
$$

where $A(z), B(z)$ are two univariate polynomials of $z=e^{i \xi}$ satisfying some conditions (see [13]). The high-pass filter coefficients $d_{\mathbf{n}}$ are given by

$$
d_{\mathbf{n}}=(-1)^{n_{1}} c_{\mathbf{e}-\mathbf{n}},
$$

where $\mathbf{n}=\left(n_{1}, n_{2}\right) \in \mathbb{Z}^{2}, \mathbf{e}=(1,0)$, and $c_{\mathbf{n}}$ are low-pass filter coefficients.

Lai [18] modified the Belogay and Wang method to construct nonseparable wavelets using the dilation matrix $\left[\begin{array}{ll}2 & 0 \\ 0 & 2\end{array}\right]$. According to the Lai method, their filter bank has the form

$$
\begin{aligned}
P_{0}\left(z_{1}, z_{2}\right)= & {\left[A\left(z_{1}\right)+z_{2} B\left(z_{1}\right)\right]\left[A\left(z_{2}\right)+z_{1}^{2} B\left(z_{2}\right)\right], } \\
P_{1}\left(z_{1}, z_{2}\right)= & z_{2}\left[A\left(z_{1}\right)+z_{2} B\left(z_{1}\right)\right]\left[A\left(-z_{2}^{-1}\right)+z_{1}^{2} B\left(-z_{2}^{-1}\right)\right], \\
P_{2}\left(z_{1}, z_{2}\right)= & z_{1}\left[A\left(-z_{1}^{-1}\right)+z_{2}^{-1} B\left(-z_{1}^{-1}\right)\right] \\
& \times\left[A\left(z_{2}\right)+z_{1}^{2} B\left(z_{2}\right)\right], \\
P_{3}\left(z_{1}, z_{2}\right)= & z_{1} z_{2}\left[A\left(-z_{1}^{-1}\right)+z_{2}^{-1} B\left(-z_{1}^{-1}\right)\right] \\
& \times\left[A\left(-z_{2}^{-1}\right)+z_{1}^{2} B\left(-z_{2}^{-1}\right)\right],
\end{aligned}
$$

where $A(z), B(z)$ are two univariate polynomials of $z=e^{i \xi}$ satisfying some conditions, $z_{1}=e^{i \xi}$, and $z_{2}=e^{i \eta}$ (see [18]). 
Ayache [11, 12] proposed two methods for constructing bivariate compactly supported orthogonal wavelets using the dilation matrix $\left[\begin{array}{ll}2 & 0 \\ 0 & 2\end{array}\right]$. Let $\alpha(x), \beta(x)$ be two univariate trigonometric polynomials, such that

$$
\alpha(0)=1, \quad|\alpha(x)|^{2}+|\beta(x)|^{2}=1 .
$$

Then according to one of the two methods, the nonseparable filter banks have the form

$$
\begin{aligned}
& P_{0}\left(z_{1}, z_{2}\right)=u_{0}\left(z_{1}\right)\left[\alpha(2 \eta) v_{0}\left(z_{2}\right)+\beta(2 \eta) v_{1}\left(z_{2}\right) z_{1}^{-2}\right], \\
& P_{1}\left(z_{1}, z_{2}\right)=u_{0}\left(z_{1}\right)\left[\overline{\beta(2 \eta)} v_{0}\left(z_{2}\right)-\overline{\alpha(2 \eta)} v_{1}\left(z_{2}\right) z_{1}^{-2}\right], \\
& P_{2}\left(z_{1}, z_{2}\right)=u_{1}\left(z_{1}\right)\left[\alpha(2 \eta) v_{0}\left(z_{2}\right)+\beta(2 \eta) v_{1}\left(z_{2}\right) z_{1}^{-2}\right], \\
& P_{3}\left(z_{1}, z_{2}\right)=u_{1}\left(z_{1}\right)\left[\overline{\beta(2 \eta)} v_{0}\left(z_{2}\right)-\overline{\alpha(2 \eta)} v_{1}\left(z_{2}\right) z_{1}^{-2}\right],
\end{aligned}
$$

where $\left\{u_{k}(z)\right\}_{0 \leq k \leq 1},\left\{v_{k}(z)\right\}_{0 \leq k \leq 1}$ are two given univariate filter banks, $z_{1}=e^{i \xi}$, and $z_{2}=e^{i \eta}$.

By combining the previous three methods, we propose, in the following, a method based on a suitable choice of the univariate filter bank, together with the basic ideas of Belogay-Wang approach. In particular, we use the univariate Daubechies filter bank to define the bivariate nonseparable filter bank as follows.

Let $r$ be a nonnegative integer, and let $\left\{m_{i}(z)\right\}_{0 \leq i \leq 1}$ be the univariate Daubechies filter bank $[2,27]$ with accuracy $r+1$; that is,

$$
m_{0}(z)=\left(\frac{1+z}{2}\right)^{r+1} H_{r+1}(z), \quad m_{1}(z)=e^{-i \xi} m_{0}\left(-z^{-1}\right),
$$

where

$$
\left|H_{r+1}(z)\right|^{2}:=\sum_{k=0}^{r}\left(\begin{array}{c}
r+k \\
k
\end{array}\right)\left(\sin ^{2} \frac{\xi}{2}\right)^{k}
$$

and $z=e^{i \xi}$. Let $A(z), B(z)$ be two univariate polynomials of $z=e^{i \xi}$ with real coefficients, and let $A(1) \neq 0, B(0) \neq 0$. We define four bivariate polynomials of $z_{1}=e^{i \xi}, z_{2}=e^{i \eta}$ which are the forms of the filter banks of this paper, as follows:

$$
\begin{aligned}
& P_{0}\left(z_{1}, z_{2}\right):=m_{0}\left(z_{2}\right)\left[A\left(z_{1}\right)+z_{2}^{2} B\left(z_{1}\right)\right], \\
& P_{1}\left(z_{1}, z_{2}\right):=z_{1} m_{0}\left(z_{2}\right)\left[B\left(-z_{1}^{-1}\right)+z_{2}^{2} A\left(-z_{1}^{-1}\right)\right], \\
& P_{2}\left(z_{1}, z_{2}\right):=m_{1}\left(z_{2}\right)\left[A\left(z_{1}\right)+z_{2}^{2} B\left(z_{1}\right)\right], \\
& P_{3}\left(z_{1}, z_{2}\right):=z_{1} m_{1}\left(z_{2}\right)\left[B\left(-z_{1}^{-1}\right)+z_{2}^{2} A\left(-z_{1}^{-1}\right)\right] .
\end{aligned}
$$

Let $r$ be a nonnegative integer, and let $v$ be an odd integer with $v \geq \operatorname{deg} A(z)$. Now, we define the polynomials $A(z)$ and $B(z)$ of $z=e^{i \xi}$ as follows:

$$
\begin{gathered}
A(z):=z^{\nu}\left(\frac{1+z^{-1}}{2}\right)^{r} L\left(z^{-1}\right) S\left(z^{-2}\right), \\
B(z):=\left(\frac{1+z}{2}\right)^{r}\left(\frac{1-z}{2}\right)^{2 r} L(-z) Q\left(z^{2}\right),
\end{gathered}
$$

where $L(z), S(z)$, and $Q(z)$ are given

by

$$
\begin{gathered}
\left|S\left(z^{2}\right)\right|^{2}=1-\left(\frac{1-z^{2}}{4}\right)^{r}\left(\frac{1-z^{-2}}{4}\right){ }^{r}\left|Q\left(z^{2}\right)\right|^{2}, \\
|L(z)|^{2}=\sum_{k=0}^{r-1}\left(\begin{array}{c}
r+k-1 \\
k
\end{array}\right)\left(\frac{1-u}{2}\right)^{k}+(1-u)^{r} u R\left(u^{2}\right), \\
u:=\frac{1}{2}\left(z+z^{-1}\right), \\
S(1)=L(1)=1, \quad Q(1)=(-1)^{r} L(-1), \\
L(0) Q(0) \neq 0,
\end{gathered}
$$

where $R(z), Q(z)$ are two arbitrary polynomials and $Q(z)$ satisfies (37).

The following condition (to be used in the following) is given for the minimal degree of $A(z)$ and $B(z)$ :

$$
\begin{gathered}
Q(z)=\text { constant }=(-1)^{r} L(-1), \\
R(z)=\text { constant }=0, \quad v=4 r-1 .
\end{gathered}
$$

Remark 2. Obviously, the polynomial $L(z)$ defined by (36) satisfies

$$
\left|\frac{1+z}{2}\right|^{2 r}|L(z)|^{2}+\left|\frac{1-z}{2}\right|^{2 r}|L(-z)|^{2}=1
$$

This equation was solved by Daubechies in [27]. If (38) holds, then $L(z)=H_{r}(z)$.

Lemma 3. The polynomial $P_{0}\left(z_{1}, z_{2}\right)$ given by (30), where $m_{0}(z)$ is the univariate Daubechies filer defined by (28), satisfies (16) if and only if the polynomials $A(z), B(z)$ in (30) satisfy

$$
\begin{gathered}
|A(z)|^{2}+|A(-z)|^{2}+|B(z)|^{2}+|B(-z)|^{2}=1, \\
A\left(z^{-1}\right) B(z)+A\left(-z^{-1}\right) B(-z)=0 .
\end{gathered}
$$

Proof. Note that

$$
\begin{aligned}
\left|P_{0}\left(z_{1}, z_{2}\right)\right|^{2}= & \left|m_{0}\left(z_{2}\right)\right|^{2} \\
& \times\left[\left|A\left(z_{1}\right)\right|^{2}+\left|B\left(z_{1}\right)\right|^{2}+z_{2}^{-2} A\left(z_{1}\right) B\left(z_{1}^{-1}\right)\right. \\
& \left.+z_{2}^{2} A\left(z_{1}^{-1}\right) B\left(z_{1}\right)\right], \\
& \left|m_{0}(z)\right|^{2}+\left|m_{0}(-z)\right|^{2}=1 .
\end{aligned}
$$


If (40) and (41) hold, we have

$$
\begin{gathered}
\sum_{k_{1}, k_{2} \in\{0,1\} \times\{0,1\}}\left|P_{0}\left((-1)^{k_{1}} z_{1},(-1)^{k_{2}} z_{2}\right)\right|^{2} \\
=\left|m_{0}\left(z_{2}\right)\right|^{2}\left[\left|A\left(z_{1}\right)\right|^{2}+\left|B\left(z_{1}\right)\right|^{2}\right. \\
+\left|A\left(-z_{1}\right)\right|^{2}+\left|B\left(-z_{1}\right)\right|^{2}+z_{2}^{-2} \\
\times\left(A\left(z_{1}\right) B\left(z_{1}^{-1}\right)+A\left(-z_{1}\right) B\left(-z_{1}^{-1}\right)\right) \\
\left.+z_{2}^{2}\left(A\left(z_{1}^{-1}\right) B\left(z_{1}\right)+A\left(-z_{1}^{-1}\right) B\left(-z_{1}\right)\right)\right] \\
+\left|m_{0}\left(-z_{2}\right)\right|^{2}\left[\left|A\left(z_{1}\right)\right|^{2}+\left|B\left(z_{1}\right)\right|^{2}\left|A\left(-z_{1}\right)\right|^{2}\right. \\
+\left|B\left(-z_{1}\right)\right|^{2}+z_{2}^{-2} \\
\times\left(A\left(z_{1}\right) B\left(z_{1}^{-1}\right)+A\left(-z_{1}\right) B\left(-z_{1}^{-1}\right)\right) \\
+z_{2}^{2}\left(A\left(z_{1}^{-1}\right) B\left(z_{1}\right)\right. \\
\left.\left.+A\left(-z_{1}^{-1}\right) B\left(-z_{1}\right)\right)\right] \\
=\left|m_{0}\left(z_{2}\right)\right|^{2}+\left|m_{0}\left(-z_{2}\right)\right|^{2}=1 .
\end{gathered}
$$

Hence (16) holds.

Conversely, if (16) holds, we have

$$
\begin{aligned}
& \sum_{k_{1}, k_{2} \in\{0,1\} \times\{0,1\}}\left|P_{0}\left((-1)^{k_{1}} z_{1},(-1)^{k_{2}} z_{2}\right)\right|^{2} \\
& =\left[\left|m_{0}\left(z_{2}\right)\right|^{2}+\left|m_{0}\left(-z_{2}\right)\right|^{2}\right] \\
& \quad \times\left[\left|A\left(z_{1}\right)\right|^{2}+\left|B\left(z_{1}\right)\right|^{2}+z_{2}^{-2} A\left(z_{1}\right) B\left(z_{1}^{-1}\right)\right. \\
& \left.\quad+z_{2}^{2} A\left(z_{1}^{-1}\right) B\left(z_{1}^{-1}\right)\right]+\left[\left|m_{0}\left(z_{2}\right)\right|^{2}+\left|m_{0}\left(-z_{2}\right)\right|^{2}\right] \\
& \quad \times\left[\left|A\left(-z_{1}\right)\right|^{2}+\left|B\left(-z_{1}\right)\right|^{2}+z_{2}^{-2} A\left(-z_{1}\right) B\left(-z_{1}^{-1}\right)\right. \\
& \left.\quad+z_{2}^{2} A\left(-z_{1}^{-1}\right) B\left(-z_{1}^{-1}\right)\right] \\
& =\left|A\left(z_{1}\right)\right|^{2}+\left|B\left(z_{1}\right)\right|^{2}+\left|A\left(-z_{1}\right)\right|^{2}+\left|B\left(-z_{1}\right)\right|^{2} \\
& +z_{2}^{-2}\left[A\left(z_{1}\right) B\left(z_{1}^{-1}\right)+A\left(-z_{1}\right) B\left(-z_{1}^{-1}\right)\right] \\
& \quad \times z_{2}^{2}\left[A\left(z_{1}^{-1}\right) B\left(z_{1}\right)+A\left(-z_{1}^{-1}\right) B\left(-z_{1}\right)\right]=1
\end{aligned}
$$

for all $\left|z_{1}\right|=1$ and $\left|z_{2}\right|=1$. Hence (40) and (41) hold.

Remark 4. Condition (41) means that $A\left(z^{-1}\right) B(z)$ contains no even powers of $z$. Therefore, $\operatorname{deg} A(z)$ must be odd if $B(0) \neq 0$.

Lemma 5. The following conditions are equivalent:

(i) the univariate real polynomials $A(z)$ and $B(z)$ satisfy condition (41) and $B(0) \neq 0$; (ii) there exist an odd integer number $v \geq \operatorname{deg} A(z)$ and real polynomials $s(z), q(z)$, and $l(z)$, such that $z^{\nu} A\left(z^{-1}\right)=$ $s\left(z^{2}\right) l(z), B(z)=q\left(z^{2}\right) l(-z)$, and $\operatorname{gcd}(l(z), l(-z))=1$.

Proof. See the proof of Lemmas 3.2 and 3.3 of [13].

Lemma 6. Let the polynomials $A(z)$ and $B(z)$ be given by (34)-(38) for $z=e^{i \xi}$; then

$$
A\left(e^{i \xi}\right)+e^{2 i \eta} B\left(e^{i \xi}\right)=0
$$

if and only if $\xi \equiv \pi(\bmod 2 \pi)$.

Proof. See the proof of Lemma 4.1 and Lemma 4.7 of [13].

By collecting the previous results and definition we can easily show the main result of this paper that is as follows.

Theorem 7 (existence). Let $r$ and $\nu$ be, respectively, a nonnegative integer and an odd integer number with $v \geq \operatorname{deg} A(z)$; let the polynomials $A(z)$ and $B(z)$ be given by (34)-(37); let the filter $m_{0}(z)$ be given by (28); let the polynomials $P_{0}\left(z_{1}, z_{2}\right)$, $P_{1}\left(z_{1}, z_{2}\right), P_{2}\left(z_{1}, z_{2}\right)$, and $P_{3}\left(z_{1}, z_{2}\right)$ be defined by (30)-(33). Then $\left\{P_{k}\left(z_{1}, z_{2}\right)\right\}_{0 \leq k \leq 3}$ is a bivariate nonseparable filter bank satisfying (16) and (21). If, in addition, the polynomials $A(z)$ and $B(z)$ satisfy condition (38), then $P_{0}\left(z_{1}, z_{2}\right)$ satisfies Cohen's criterion (18), and the filter bank $\left\{P_{k}\left(z_{1}, z_{2}\right)\right\}_{0 \leq k \leq 3}$ can be used, according to (17), (22), to define bivariate nonseparable compactly supported orthogonal wavelets.

Proof. According to Lemmas 3 and 5 by letting

$$
\begin{gathered}
s\left(z^{2}\right)=S\left(z^{2}\right), \quad q\left(z^{2}\right)=\left(\frac{1-z^{2}}{2}\right)^{r} \mathrm{Q}\left(z^{2}\right), \\
l(z)=\left(\frac{1+z}{2}\right)^{r} L(z),
\end{gathered}
$$

in order to prove that $P_{0}\left(z_{1}, z_{2}\right)$ satisfies (16), we need only to show that $A(z), B(z)$ satisfy (40). Taking into account (34) and (35), we have

$$
\begin{aligned}
|A(z)|^{2}+|B(-z)|^{2} & \left|\frac{1+z}{2}\right|^{2 r}|L(z)|^{2}\left|S\left(z^{2}\right)\right|^{2} \\
& +\left|\frac{1-z}{2}\right|^{2 r}\left|\frac{1+z}{2}\right|^{4 r}|L(z)|^{2}\left|Q\left(z^{2}\right)\right|^{2} \\
= & \left|\frac{1+z}{2}\right|^{2 r}|L(z)|^{2}\left(\left|S\left(z^{2}\right)\right|^{2}+\left|\frac{1-z}{2}\right|^{2 r}\left|\frac{1+z}{2}\right|^{2 r}\left|Q\left(z^{2}\right)\right|^{2}\right) \\
= & \left|\frac{1+z}{2}\right|^{2 r}|L(z)|^{2} .
\end{aligned}
$$

Hence, (40) follows from (39).

By a simple calculation, we can also get that $\left\{P_{k}\left(z_{1}\right.\right.$, $\left.\left.z_{2}\right)\right\}_{0 \leq k \leq 3}$ satisfies (21). In fact, let us assume that

$$
P_{0}\left(z_{1}, z_{2}\right)=u\left(z_{1}\right) v\left(z_{2}\right),
$$


where $u(z)$ and $v(z)$ are univariate low-pass filters. Then we have

$$
P_{0}\left(1, z_{2}\right)=v\left(z_{2}\right)=m_{0}\left(z_{2}\right)
$$

This leads to the contradiction that

$$
u\left(z_{1}\right)=A\left(z_{1}\right)+z_{2}^{2} B\left(z_{1}\right) .
$$

Hence $P_{0}\left(z_{1}, z_{2}\right)$ is nonseparable.

Finally, suppose that $A(z)$ and $B(z)$ satisfy (34)-(38). Let $\Omega=[-\pi, \pi] \times[-\pi, \pi]$, then $\Omega$ is a compact fundamental domain of the lattice $2 \pi \mathbb{Z}^{2}$. When $j \geq 1$ and $(\xi, \eta) \in \Omega$, it is $\left(\frac{\xi}{2^{j}}, \frac{\eta}{2^{j}}\right) \in\left[-\frac{\pi}{2}, \frac{\pi}{2}\right]^{2}$, so that we have

$$
A\left(e^{i \xi / 2^{j}}\right)+e^{2 i \eta / 2^{j}} B\left(e^{i \xi / 2^{j}}\right) \neq 0, \quad m_{0}\left(e^{i \eta / 2^{j}}\right) \neq 0
$$

for all $j \geq 1$ and $(\xi, \eta) \in \Omega$ by using Lemma 6 and the result of [27]. Hence $P_{0}\left(z_{1}, z_{2}\right)$ satisfies Cohen's criterion (18).

3.2. Accuracy. We will use the same method as given in [13] to study the accuracy of our scaling function. Let recall its definition and how the accuracy of the scaling function implies some restrictions on the low-pass filter.

According to the result of [14], an orthogonal scaling function $\varphi(\mathbf{x})$ with the dilation matrix $\left[\begin{array}{ll}2 & 0 \\ 0 & 2\end{array}\right]$ has accuracy $r+1$ if and only if its low-pass filter $P_{0}\left(z_{1}, z_{2}\right)$ satisfies the following accuracy condition:

$$
\begin{aligned}
& \left.\frac{\partial^{i+j}}{\partial z_{1}^{i} \partial z_{2}^{j}} P_{0}\left(z_{1}, z_{2}\right)\right|_{z_{1}=-1, z_{2}=1}=0, \\
& \left.\frac{\partial^{i+j}}{\partial z_{1}^{i} \partial z_{2}^{j}} P_{0}\left(z_{1}, z_{2}\right)\right|_{z_{1}=1, z_{2}=-1}=0, \\
& \left.\frac{\partial^{i+j}}{\partial z_{1}^{i} \partial z_{2}^{j}} P_{0}\left(z_{1}, z_{2}\right)\right|_{z_{1}=-1, z_{2}=-1}=0,
\end{aligned}
$$

for all $i, j \geq 0$ with $i+j \leq r$. Therefore, when $P_{0}\left(z_{1}, z_{2}\right)$ satisfies (52), we say that it has accuracy $r+1$.

Lemma 8. Let the polynomials $m_{0}(z)$ and $P_{0}\left(z_{1}, z_{2}\right)$ be given by (28) and (30), respectively; then $P_{0}\left(z_{1}, z_{2}\right)$ satisfies (52) if and only if the polynomials $A(z), B(z)$ in (30) defined by (34) have the form

$$
A(z)=\left(\frac{1+z}{2}\right)^{r} A_{0}(z), \quad B(z)=\left(\frac{1+z}{2}\right)^{r} B_{0}(z),
$$

where $A_{0}(z), B_{0}(z)$ are polynomials with real coefficients satisfying

$$
A_{0}(-1)+B_{0}(-1)=0 \text {. }
$$

Proof. It is easy to check that

$$
\begin{aligned}
\frac{\partial^{i} P_{0}\left(z_{1}, z_{2}\right)}{\partial z_{1}^{i}}= & m_{0}\left(z_{2}\right)\left[A^{(i)}\left(z_{1}\right)+z_{2}^{2} B^{(i)}\left(z_{1}\right)\right], \\
\frac{\partial^{i+1} P_{0}\left(z_{1}, z_{2}\right)}{\partial z_{1}^{i} \partial z_{2}}= & m_{0}^{\prime}\left(z_{2}\right)\left[A^{(i)}\left(z_{1}\right)+z_{2}^{2} B^{(i)}\left(z_{1}\right)\right] \\
& +2 z_{2} m_{0}\left(z_{2}\right) B^{(i)}\left(z_{1}\right), \\
& \vdots \\
\frac{\partial^{i+j} P_{0}\left(z_{1}, z_{2}\right)}{\partial z_{1}^{i} \partial z_{2}^{j}=} & m_{0}^{(j)}\left(z_{2}\right)\left[A^{(i)}\left(z_{1}\right)+z_{2}^{2} B^{(i)}\left(z_{1}\right)\right] \\
& +2 j z_{2} m_{0}^{(j-1)}\left(z_{2}\right) B^{(i)}\left(z_{1}\right) \\
& +j(j-1) m_{0}^{(j-2)}\left(z_{2}\right) B^{(i)}\left(z_{1}\right),
\end{aligned}
$$

for all $i \geq 0$ and $j \geq 2$.

If $A(z), B(z)$ satisfy (53) and (54), it is not difficult to show that $P_{0}\left(z_{1}, z_{2}\right)$ satisfies also (52). On the other hand, if $P_{0}\left(z_{1}, z_{2}\right)$ satisfies (52) and noting that $m_{0}(1)=1$, we have

$$
\begin{gathered}
A^{(i)}(-1)+B^{(i)}(-1)=0, \quad \text { for } 0 \leq i \leq r, \\
A^{(i)}(-1)=0, \quad B^{(i)}(-1)=0, \quad \text { for } 0 \leq i<r .
\end{gathered}
$$

So that $z=-1$ is a root of multiplicity $r$ of $A(z), B(z)$. Hence $A(z), B(z)$ have the form of $(53)$, and $A^{(r)}(-1)+B^{(r)}(-1)=0$ yields (54).

Lemma 9. Let the polynomials $m_{0}(z)$ and $P_{0}\left(z_{1}, z_{2}\right)$ be given by (28) and (30), respectively, and assume also that $A(z)$ and $B(z)$ in (30) are polynomials with $A(1) \neq 0$ and $B(0) \neq 0$. Then $P_{0}\left(z_{1}, z_{2}\right)$ satisfies (41) with accuracy $r+1$ if and only if there exist an odd integer number $v \geq \operatorname{deg} A(z)$ and real polynomials $S(z), Q(z)$, and $L(z)$, such that $A(z)$ and $B(z)$ satisfy (34) and (37).

Proof. If there exist an odd integer $v \geq \operatorname{deg} A(z)$ and real polynomials $S(z), Q(z)$, and $L(z)$ such that $A(z)$ and $B(z)$ satisfy (34), and (37), then $P_{0}\left(z_{1}, z_{2}\right)$ satisfies (41) and has accuracy $r+1$ by Lemmas 5 and 8 by letting

$$
\begin{gathered}
s\left(z^{2}\right)=S\left(z^{2}\right), \quad q\left(z^{2}\right)=\left(\frac{1-z^{2}}{2}\right)^{r} \mathrm{Q}\left(z^{2}\right), \\
l\left(z^{2}\right)=\left(\frac{1+z}{2}\right)^{r} L(z), \\
A_{0}(z)=z^{\nu-r} L\left(z^{-1}\right) S\left(z^{-2}\right) \\
B_{0}(z)=L(-z)\left(\frac{1-z}{2}\right)^{2 r} \mathrm{Q}\left(z^{2}\right) .
\end{gathered}
$$

Now, suppose that $P_{0}\left(z_{1}, z_{2}\right)$ satisfies (41) with accuracy $r+1$. By Lemma $8, z=-1$ is a root of multiplicity $2 r$ of $G(z):=$ $z^{\nu} A\left(z^{-1}\right) B(z)$. Since $G(-z)=G(z)$ by $(41), z=1$ is another 
root of multiplicity $2 r$ of $G(z)$. But $A(1) \neq 0$, so $z=1$ is a root of multiplicity $2 r$ of $B(z)$. Hence, we have

$$
\begin{gathered}
A\left(z^{-1}\right)=\left(\frac{1+z}{2}\right)^{r} A_{1}\left(z^{-1}\right), \\
B(z)=\left(\frac{1+z}{2}\right)^{r}\left(\frac{1-z}{2}\right)^{2 r} B_{1}(z) .
\end{gathered}
$$

By substituting (58) in (41), we have

$$
A_{1}\left(z^{-1}\right) B_{1}(z)+A_{1}\left(-z^{-1}\right) B_{1}(-z)=0 .
$$

Then, by applying Lemma 5 to $A_{1}(z)$ and $B_{1}(z)$, we get that there exist an odd integer $v \geq \operatorname{deg} A(z)$ and real polynomials $S(z), Q(z)$, and $L(z)$, such that (34) hold.

Since $B(1)=0$, and therefore $A(1)=1$, we can normalize $S(z)$ and $L(z)$, so that $S(1)=L(1)=1$. And $Q(1)=$ $(-1)^{r} L(-1)$ follows from condition (54). Hence $A(z)$ and $B(z)$ satisfy (34), and (37).

Theorem 10 (accuracy). Let the polynomials $A(z), B(z)$, and $P_{0}\left(z_{1}, z_{2}\right)$ be given by (34)-(38) and (30) with $m_{0}\left(z_{2}\right)$ according to (28). Then $P_{0}\left(z_{1}, z_{2}\right)$ is a low-pass filter which can generate bivariate nonseparable compactly supported orthogonal scaling function with accuracy $r+1$.

Proof. This theorem follows from Lemmas 8, 9, and Theorem 7.

Remark 11. (i) According to the proof of Theorem 2.1 of [13], if $A(z), B(z)$ satisfy (40), the polynomials $S(z)$ and $L(z)$ in Lemma 9 must satisfy (35) and (36).

(ii) The existence of $S(z)$ and $L(z)$ satisfying (35)-(38) is ensured by Corollary 4.2 of [13].

3.3. Smoothness. In this section, we measure the smoothness of the scaling function $\varphi(\mathbf{x})$ generated by $P_{0}\left(z_{1}, z_{2}\right)$ by its Hölder exponent (see $[13,23])$. Let $s=n+t$, where $n$ is a nonnegative integer and $0 \leq t<1$. For nonnegative integers $\alpha_{1}, \alpha_{2}$, and $\alpha=\alpha_{1}+\alpha_{2}, D^{\alpha} \varphi(\mathbf{x})$ denotes the partial derivative of $\varphi(\mathbf{x})$; that is,

$$
D^{\alpha} \varphi(\mathbf{x}):=\frac{\partial^{\alpha} \varphi(\mathbf{x})}{\partial^{\alpha_{1}} x_{1} \partial^{\alpha_{2}} x_{2}}, \quad \mathbf{x}=\left(x_{1}, x_{2}\right) \in \mathbb{R}^{2} .
$$

If there exists a constant $c$, such that

$$
\left|D^{\alpha} \varphi(\mathbf{x})-D^{\alpha} \varphi(\mathbf{y})\right| \leq c|\mathbf{x}-\mathbf{y}|^{t}, \quad \forall \alpha \leq n \text { and } \mathbf{x}, \mathbf{y} \in \mathbb{R}^{2}
$$

we say that $\varphi(\mathbf{x}) \in C^{s}\left(\mathbb{R}^{2}\right)$. It is well known that if the Fourier transform $\widehat{\varphi}(\omega)$ of $\varphi(\mathbf{x})$ satisfies

$$
|\widehat{\varphi}(\omega)| \leq c(1+|\xi|)^{-s-1-\varepsilon}(1+|\eta|)^{-s-1-\varepsilon}, \quad \omega=(\xi, \eta) \in \mathbb{R}^{2}
$$

for some constants $c>0, \varepsilon>0$, then $\varphi(\mathbf{x}) \in C^{s}\left(\mathbb{R}^{2}\right)$ (see $[13,23])$.
TABLE 1: The coefficients of the polynomials $A(z)$ and $B(z)$ of the example given in Section 3.4

\begin{tabular}{lc}
\hline$k$ & $a_{k}, b_{k}$ \\
\hline 0 & $0.007903570868,0.036969146934$ \\
1 & $-0.013689386305,-0.064032440902$ \\
2 & $-0.138084015734,-0.056780853066$ \\
3 & $0.121182418797,0.137970734671$ \\
4 & $0.549926093940,0.002654265329$ \\
5 & $0.346172096397,-0.083844146934$ \\
6 & $0.080254350926,0.017157440802$ \\
7 & $0.046334871111,0.009905853066$ \\
\hline
\end{tabular}

Let us assume that the polynomials $A(z)$ and $B(z)$ satisfying (34)-(38) are

$$
\begin{aligned}
& A(z)=\left(\frac{1+z}{2}\right)^{r} A_{0}(z), \\
& B(z)=\left(\frac{1+z}{2}\right)^{r} B_{0}(z),
\end{aligned}
$$

and let

$$
l(z)=\left|A_{0}(z)\right|+\left|B_{0}(z)\right| .
$$

Define a polynomial $T_{r}(y)$ as follows:

$$
T_{r}(y)=\sum_{k=0}^{r-1}\left(\begin{array}{c}
r+k-1 \\
k
\end{array}\right) y^{k}, \quad \text { for } y \in \mathbb{R} .
$$

Lemma 12. Let $l(z)$ be given by (64). Then there exist constants $c>0$ and $0<\varepsilon<1$, such that

$$
\prod_{k=1}^{\infty} l\left(z^{2^{-k}}\right) \leq c|\xi|^{\mu_{r}+1-\varepsilon}
$$

where $z=e^{i \xi}$ and $\mu_{r}:=(1 / 2) \log _{2} T_{r}(3 / 4)$. Furthermore, it is

$$
\begin{gathered}
r-\mu_{r}>\left(1-\frac{1}{2} \log _{2} 3\right) r+\frac{1}{2} \log _{2} 3, \\
\lim _{r \rightarrow \infty} \frac{r-\mu_{r}-2}{r}=1-\frac{1}{2} \log _{2} 3 \approx 0.2075 .
\end{gathered}
$$

Proof. See the proof of Lemma 4.1, 4.3-4.5 of [13].

Theorem 13 (smoothness). Let the polynomials $m_{0}(z), A(z)$, and $B(z)$ be given by (28) and (34)-(38), respectively. If $\varphi(\mathbf{x})$ is the bivariate scaling function generated by the low-pass filter $P_{0}\left(z_{1}, z_{2}\right)$ according to (30) and $r \geq 5$, then $\varphi(\mathbf{x}) \in$ $C^{r-\mu_{r}-2}\left(\mathbb{R}^{2}\right)$.

Proof. We only need to prove that the Fourier transform $\widehat{\varphi}(\omega)$ of $\varphi(\mathbf{x})$ satisfies (62). In fact, by applying Lemmas 7.1.1-7.1.8 of [2], we have

$$
\left|\prod_{k=1}^{\infty} m_{0}\left(e^{i \eta / 2^{k}}\right)\right| \leq c(1+|\eta|)^{-r+\mu_{r}} \leq c(1+|\eta|)^{-r+\mu_{r}+1-\varepsilon},
$$



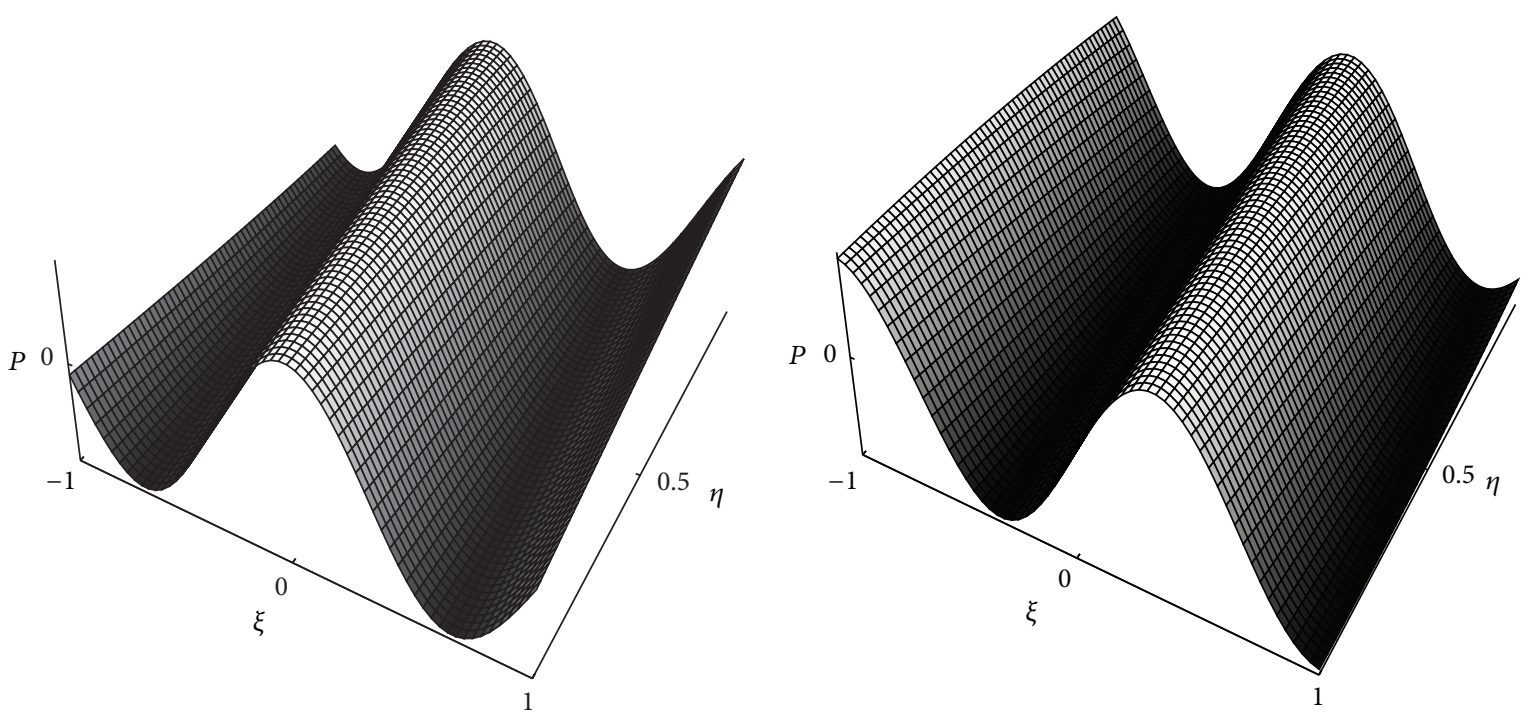

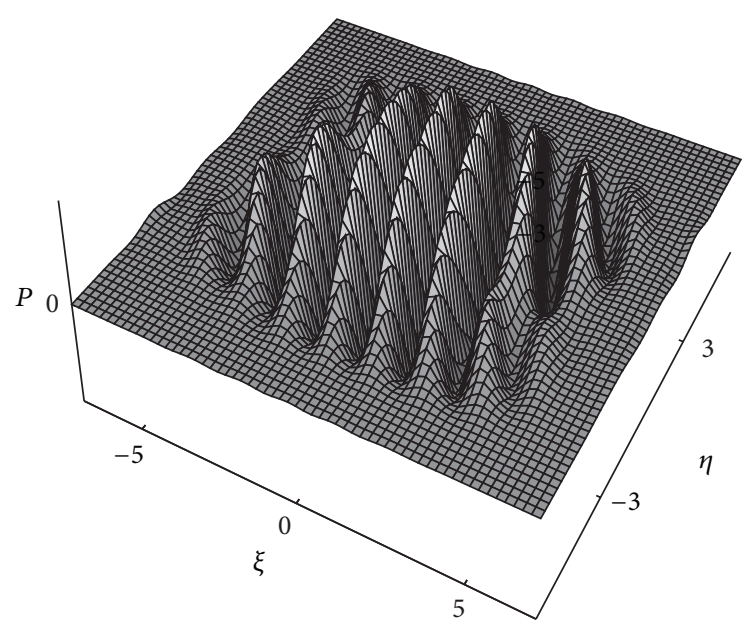

(a)

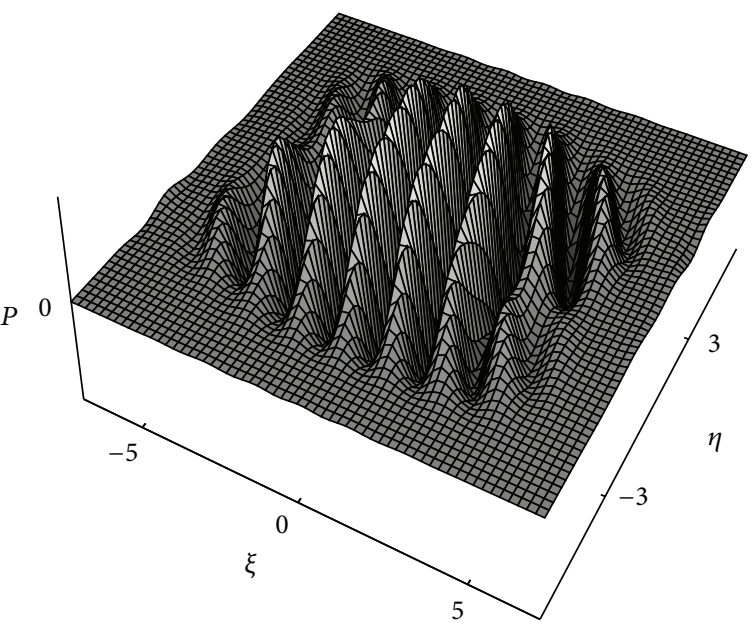

(b)

FIGURE 1: Plot of the real (a) and imaginary (b) parts of the bivariate compactly supported low-pass filter (74).

for the same $\varepsilon$ as Lemma 12 , and

$$
\left|\prod_{k=1}^{\infty}\left(\frac{1+e^{i \xi / 2^{k}}}{2}\right)^{r}\right| \leq c(1+|\xi|)^{-r} .
$$

Thus, according to (63), (64), (68), and (69) and Lemma 12, we get

$$
\begin{aligned}
& \left|\prod_{k=1}^{\infty} P\left(e^{i \xi / 2^{k}}, e^{i \eta / 2^{k}}\right)\right| \\
& \quad \leq\left|\prod_{k=1}^{\infty} m_{0}\left(e^{\frac{i \eta}{2^{k}}}\right)\right|\left|\prod_{k=1}^{\infty}\left(\frac{1+e^{\frac{i \xi}{2^{k}}}}{2}\right)\right|\left|\prod_{k=1}^{\infty} l\left(e^{i \xi / 2^{k}}\right)\right| \\
& \quad \leq c(1+|\xi|)^{-r+\mu_{r}+1-\varepsilon}(1+|\eta|)^{-r+\mu_{r}+1-\varepsilon} .
\end{aligned}
$$

Hence by using (17) it is shown that (62) holds for $s=r-\mu_{r}-$ 2.

Remark 14. (i) The wavelets have the same smoothness as the corresponding scaling function. So we only need to study the smoothness of the scaling function.

(ii) As the remark of paper [13], (67) mean that the smoothness of the bivariate orthogonal wavelets improves asymptotically by 0.2075 when $r$ is incremented by 1 . Hence, (67) guarantees the existence of bivariate orthogonal wavelets of any desired smoothness. In particular, if $r>4$, then $\varphi(\mathbf{x}) \in C^{0}\left(\mathbb{R}^{2}\right)$, if $r>8$, then $\varphi(\mathbf{x}) \in C^{1}\left(\mathbb{R}^{2}\right)$, and if $r>12$, then $\varphi(\mathbf{x}) \in C^{2}\left(\mathbb{R}^{2}\right)$.

According to the results of this section, we can summarize the following procedure for constructing the filter banks.

Step 1. Choose a nonnegative integer $r$, and let $\nu=4 r-1$. 
TABle 2: The coefficients of the low-pass filter $P_{0}\left(z_{1}, z_{2}\right)$ of the example given in Section 3.4

\begin{tabular}{cc}
\hline$k$ & $p_{k, 0}, p_{k, 1}, p_{k, 2}, p_{k, 3}$ \\
\hline 0 & $0.002629285291,0.006377324227,0.015933220981,0.028763021603$ \\
1 & $-0.004554055712,-0.011045849577,-0.027597148268,-0.049819014795$ \\
2 & $-0.045936485868,-0.111418819867,-0.082391050025,-0.027173124419$ \\
3 & $0.040313822269,0.097781064805,0.101627868651,0.094966452370$ \\
4 & $0.182944217753,0.443730695949,0.253781634345,-0.072104378716$ \\
5 & $0.115161262724,0.279323325343,0.131304280272,-0.114390178100$ \\
6 & $0.026698259299,0.064756554347,0.042614945757,0.003008971526$ \\
7 & $0.015414247193,0.037387214084,0.024603750404,0.001737230521$ \\
\hline$k$ & $p_{k, 4}, p_{k, 5}, p_{k, 6}, p_{k, 7}$ \\
\hline 0 & $0.016325987784,-0.004712828742,-0.003158691008,0.001302285961$ \\
1 & $-0.028277440326,0.008162858829,0.005471013312,-0.002255625450$ \\
2 & $-0.014314162669,0.002801853046,0.004851428418,-0.002000178904$ \\
3 & $0.053095656592,-0.014358762363,-0.011788395329,0.004860197371$ \\
4 & $-0.045765749098,0.019013502031,-0.000226783811,0.000093499925$ \\
5 & $-0.068135421758,0.023514243110,0.007163750722,-0.002953518393$ \\
6 & $0.001033287041,0.000510619607,-0.001465953599,0.000604393018$ \\
7 & $0.000596568551,0.000294806368,-0.000846368705,0.000348946471$ \\
\hline
\end{tabular}

Step 2. Use some spectral factorization method to make a list of all polynomials $S(z)$ and $L(z)$ that satisfy (35)-(38). Substitute $S(z), L(z)$, and $Q$ in (34) to get $A(z)$ and $B(z)$.

Step 3. Construct the low-pass filter $P_{0}\left(z_{1}, z_{2}\right)$ by substituting $A(z), B(z)$, and univariate Daubechies low-pass filter $m_{0}\left(z_{2}\right)$ in $(30)$.

Step 4. Construct the high-pass filters $P_{i}\left(z_{1}, z_{2}\right), i=1,2,3$ by substituting $A(z), B(z)$, and univariate Daubechies filters $m_{0}\left(z_{2}\right)$ and $m_{1}\left(z_{2}\right)$ in $(31)-(33)$.

3.4. Example. Finally, we give an example of bivariate nonseparable compactly supported orthogonal low-pass filter by using our method.

Let $r=2$. The following polynomial $m_{0}(z)$ is the univariate Daubechies low-pass filter with accuracy 3 (see [27]):

$$
m_{0}(z)=\sum_{k=0}^{5} p_{k} z^{k}
$$

where

$$
\begin{array}{ll}
p_{0}=0.332670552950, & p_{1}=0.806891509311, \\
p_{2}=0.459877502118, & p_{3}=-0.135011020010, \\
p_{4}=-0.085441273882, & p_{5}=0.035226291886 .
\end{array}
$$

If (38) holds, then there are four polynomials $S(z)$ satisfying (35), (37) and two polynomials $L(z)$ satisfying (36),
(37). Hence, one can get 8 kinds of $A(z)$ and $B(z)$ satisfying (34)-(38) (see [13]). One of them is given as follows:

$$
A(z)=\sum_{k=0}^{7} a_{k} z^{k}, \quad B(z)=\sum_{k=0}^{7} b_{k} z^{k}
$$

where the coefficients $a_{k}, b_{k}, k=0,1, \ldots, 7$ are displayed in Table 1.

Then, by using (30), we can get a bivariate nonseparable compactly supported orthogonal low-pass filter (Figure 1) given as follows:

$$
P_{0}\left(z_{1}, z_{2}\right)=\sum_{k, l=0}^{7} p_{k, l} z_{1}^{k} z_{2}^{l}
$$

where the coefficients $p_{k, l}$ are displayed in Table 2.

For instance, by explicit computation and the approximation of coefficients the first few terms of the filter look like

$$
\begin{aligned}
& 0.002629-0.004553 e^{(i / 2) \xi}-0.045936 e^{i \xi} \\
& +0.040313 e^{(3 i / 2) \xi}+0.182944 e^{2 i \xi}+0.115161 e^{(5 i / 2) \xi} \\
& +0.026698 e^{3 i \xi}+0.015413 e^{(7 i / 2) \xi} \\
& -0.011045 e^{(i / 2) \xi+(i / 2) \eta}-0.111418 e^{i \xi+(i / 2) \eta} \\
& +0.09778 e^{(3 i / 2) \xi+(i / 2) \eta}+0.44373 e^{2 i \xi+(i / 2) \eta} \ldots .
\end{aligned}
$$




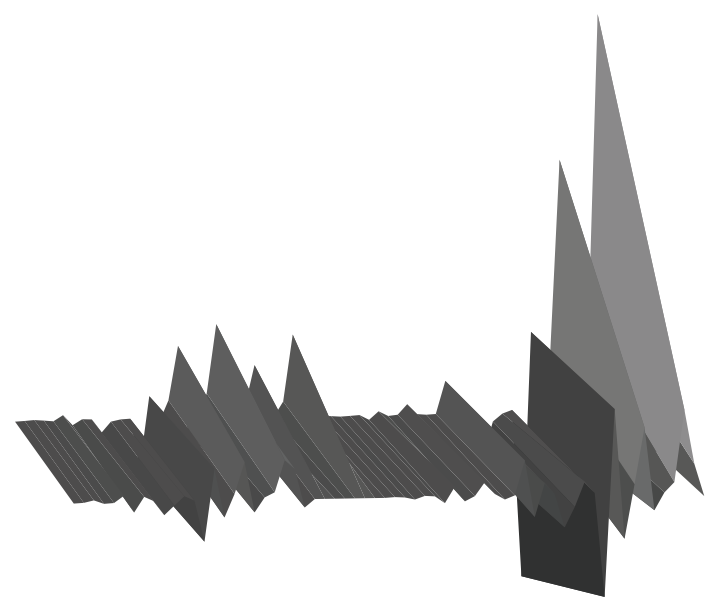

FIGURE 2: The scaling function generated by the low-pass filter (74).

By the inverse Fourier transform we finally obtain the scaling function, so that we have a sequence in the following form:

$$
\begin{aligned}
& -0.00921 \delta(-3+x) \delta(-3+y) \\
& -0.001424 \delta(-2+x) \delta(-3+y) \\
& +0.030481 \delta(-1+x) \delta(-3+y) \\
& -0.019846 \delta(x) \delta(-3+y) \\
& -0.010634 \delta(-7+2 x) \delta(-3+y) \\
& +0.090022 \delta(-5+2 x) \delta(-3+y) \\
& -0.148136 \delta(-3+2 x) \delta(-3+y) \\
& +0.06875 \delta(-1+2 x) \delta(-3+y)+\cdots
\end{aligned}
$$

The scaling function generated by the low-pass filter (Figure 2) has accuracy 3. By (31)-(33), one can easily obtain the corresponding high-pass filters. We omit it here.

\section{Conclusion}

We gave a method for constructing bivariate nonseparable compactly supported orthogonal wavelets by combining three methods using the dilation matrix $\left[\begin{array}{ll}2 & 0 \\ 0 & 2\end{array}\right]$. We studied the accuracy of the scaling functions and the smoothness of the wavelets constructed by this method. We proved that the scaling functions have the same accuracy order as the univariate Daubechies low-pass filter used in this method and the wavelets can be made arbitrarily smooth by choosing the accuracy order.

\section{Acknowledgments}

The authors wish to thank the anonymous reviewers for their valuable comments and suggestions that have improved the presentation of this paper. This work was supported by the National Natural Science Foundation of China (11271001, 61170311), 973 Program (2013CB329404), and
Sichuan Province Science and Technology Research Project (12ZC1802). a

\section{References}

[1] C. K. Chui, An Introduction to Wavelets, vol. 1 of Wavelet Analysis and Its Applications, Academic Press, Boston, Mass, USA, 1992.

[2] I. Daubechies, Ten Lectures on Wavelets, vol. 61 of CBMSNSF Regional Conference Series in Applied Mathematics, SIAM, Philadelphia, Pa, USA, 1992.

[3] A. Said and W. A. Pearlman, "A new fast and efficient image codec based on set partitioning in hierarchical trees," IEEE Transactions on Circuits and Systems for Video Technology, vol. 6, no. 3, pp. 243-250, 1996.

[4] C. Cattani and J. Rushchitsky, Wavelet and Wave Analysis as Applied to Materials with Micro or Nanostructure, vol. 74 of Series on Advances in Mathematics for Applied Sciences, World Scientific, Singapore, 2007.

[5] S. Mallat, A Wavelet Tour of Signal Processing: The Sparse Way, Academic Press, Amsterdam, The Netherlands, 3rd edition, 2008.

[6] G. Strang and T. Nguyen, Wavelets and Filter Banks, WellesleyCambridge Press, Wellesley, Mass, USA, 1996.

[7] M. Vetterli and J. Kovacevic, Wavelets and Subband Coding, Prentice Hall, New York, NY, USA, 1995.

[8] C. Cattani and L. M. S. Ruiz, "Discrete differential operators in multidimensional Haar wavelet spaces," International Journal of Mathematics and Mathematical Sciences, vol. 2004, no. 44, pp. 2347-2355, 2004.

[9] A. Cohen and I. Daubechies, "Nonseparable bidimensional wavelet bases," Revista Matemática Iberoamericana, vol. 9, no. 1, pp. 51-137, 1993.

[10] P. Wojtaszczyk, A Mathematical Introduction to Wavelets, vol. 37 of London Mathematical Society Student Texts, Cambridge University Press, Cambridge, UK, 1997.

[11] A. Ayache, "Construction of non-separable dyadic compactly supported orthonormal wavelet bases for $L^{2}\left(R^{2}\right)$ of arbitrarily high regularity," Revista Matemática Iberoamericana, vol. 15, no. 1, pp. 37-58, 1999.

[12] A. Ayache, "Some methods for constructing nonseparable, orthonormal, compactly supported wavelet bases," Applied and Computational Harmonic Analysis, vol. 10, no. 1, pp. 99-111, 2001.

[13] E. Belogay and Y. Wang, "Arbitrarily smooth orthogonal nonseparable wavelets in $R^{2}$," SIAM Journal on Mathematical Analysis, vol. 30, no. 3, pp. 678-697, 1999.

[14] C. Cabrelli, C. Heil, and U. Molter, "Accuracy of lattice translates of several multidimensional refinable functions," Journal of Approximation Theory, vol. 95, no. 1, pp. 5-52, 1998.

[15] B. Han, "Symmetric multivariate orthogonal refinable functions," Applied and Computational Harmonic Analysis, vol. 17, no. 3, pp. 277-292, 2004.

[16] W. He and M. J. Lai, "Examples of bivariate nonseparable compactly supported orthonormal continuous wavelets," IEEE Transactions on Image Processing, vol. 9, no. 5, pp. 949-953, 2000.

[17] A. Karoui, "A note on the design of nonseparable orthonormal wavelet bases of $L^{2}\left(R^{3}\right)$," Applied Mathematics Letters, vol. 18, no. 3, pp. 293-298, 2005.

[18] M. J. Lai, "Methods for constructing nonseparable compactly supported orthonormal wavelets," in Wavelet Analysis: Twenty 
Year's Development, D. X. Zhou, Ed., vol. 1, pp. 231-251, World Scientific, Singapore, 2002.

[19] M. J. Lai and D. W. Roach, "Nonseparable symmetric wavelets with short support," in 7th Wavelet Applications in Signal and Image Processing, vol. 3813 of Proceedings of SPIE, pp. 132-146, 1999.

[20] J. S. Leng, T. Z. Huang, and Y. D. Fu, "Construction of bivariate nonseparable compactly supported biorthogonal wavelets," in Proceedings of the International Conference on Machine Learning and Cybernetics, pp. 3625-3629, Kunming, China, July 2008.

[21] J. S. Leng, T. Z. Huang, Y. D. Fu, and C. H. Lai, "A method for constructing trivariate nonseparable compactly supported orthogonal wavelets," International Journal of Computer Mathematics, vol. 86, no. 7, pp. 1264-1273, 2009.

[22] J. S. Leng, T. Z. Huang, Y. F. Jing, and W. Jiang, "A study on conjugate quadrature filters," EURASIP Journal on Advances in Signal Processing, vol. 2011, Article ID 231754, 2011.

[23] R. L. Long, Multi-Dimensional Wavelet Analysis, Springer, Beijing,China, 1995.

[24] D. Stanhill and Y. Y. Zeevi, "Two dimensional orthogonal wavelets with vanishing moments," IEEE Transactions on Signal Processing, vol. 44, no. 10, pp. 2579-2590, 1996.

[25] D. Stanhill and Y. Y. Zeevi, “Two dimensional orthogonal filter banks and wavelets with linear phase," IEEE Transactions on Signal Processing, vol. 46, no. 1, pp. 183-190, 1998.

[26] L. F. Villemoes, "Continuity of nonseparable quincunx wavelets," Applied and Computational Harmonic Analysis, vol. 1, no. 2, pp. 180-187, 1994.

[27] I. Daubechies, "Orthonormal bases of compactly supported wavelets," Communications on Pure and Applied Mathematics, vol. 41, no. 7, pp. 909-996, 1988. 


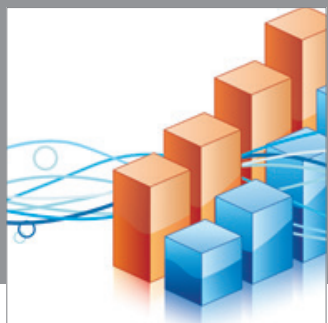

Advances in

Operations Research

mansans

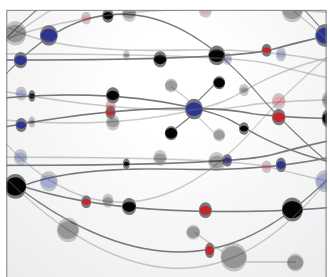

The Scientific World Journal
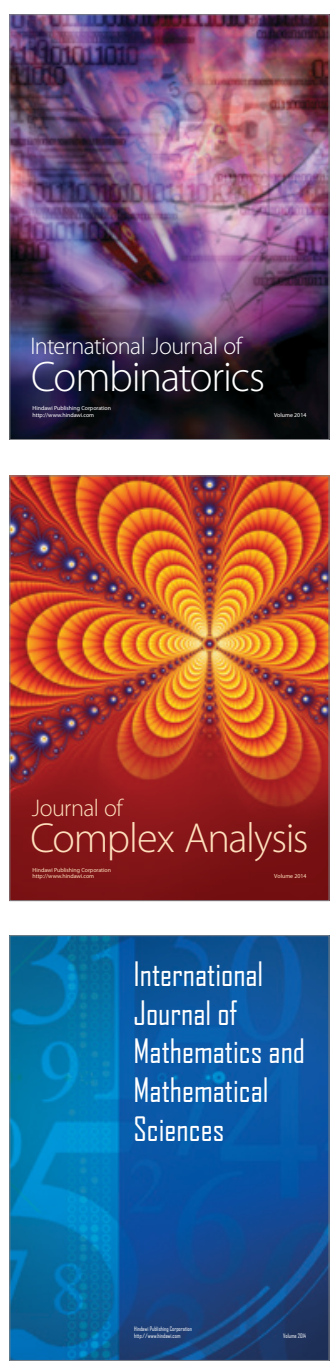
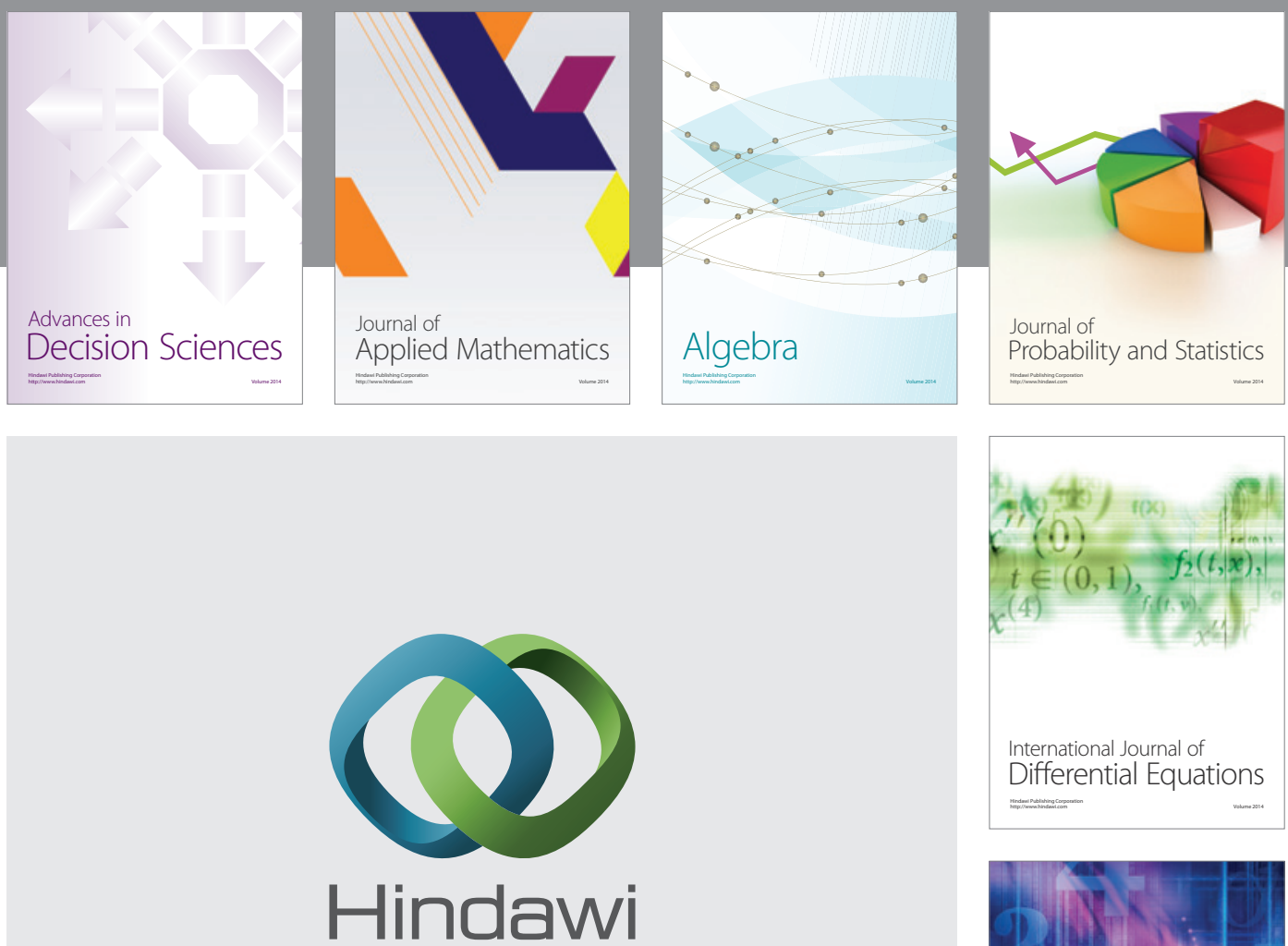

Submit your manuscripts at http://www.hindawi.com
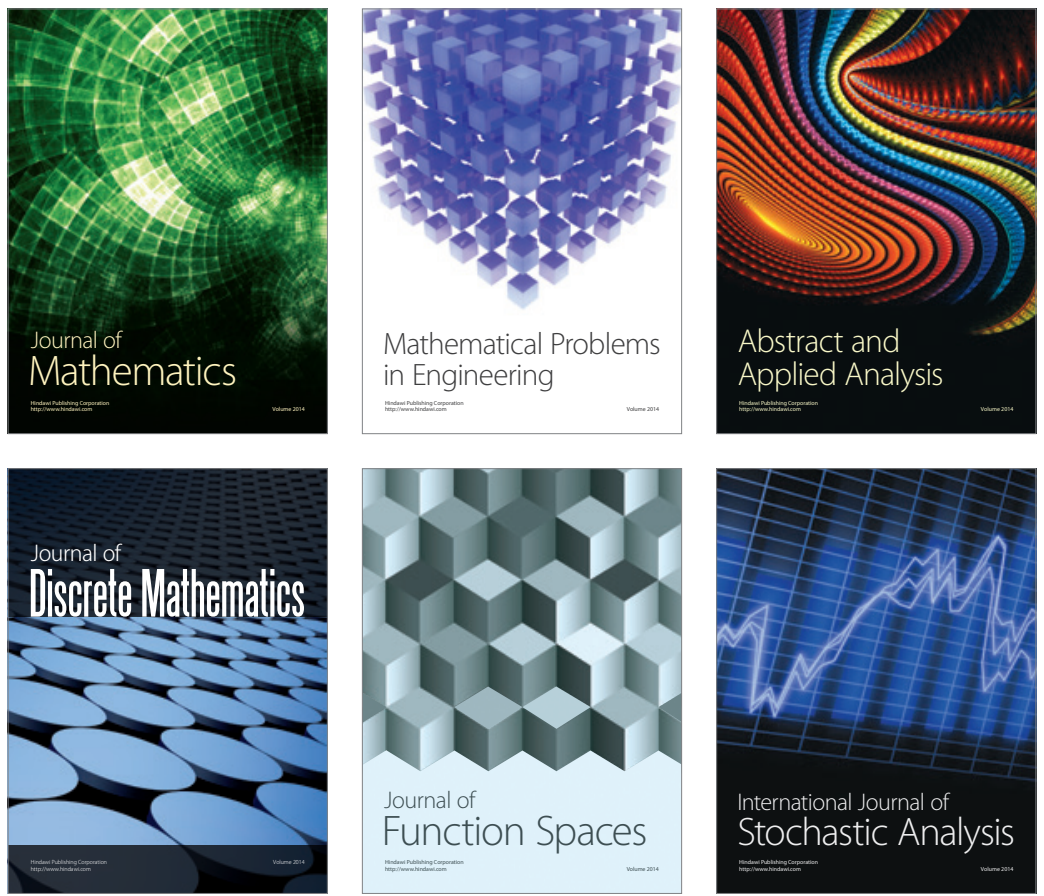

Journal of

Function Spaces

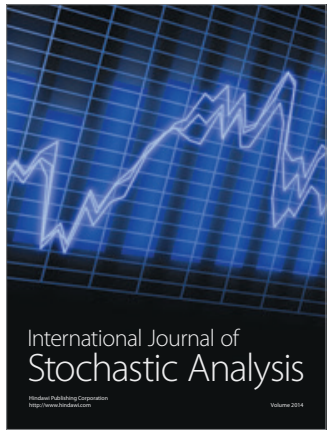

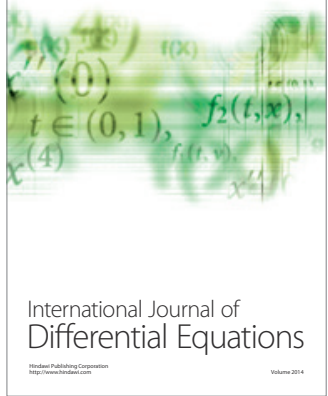
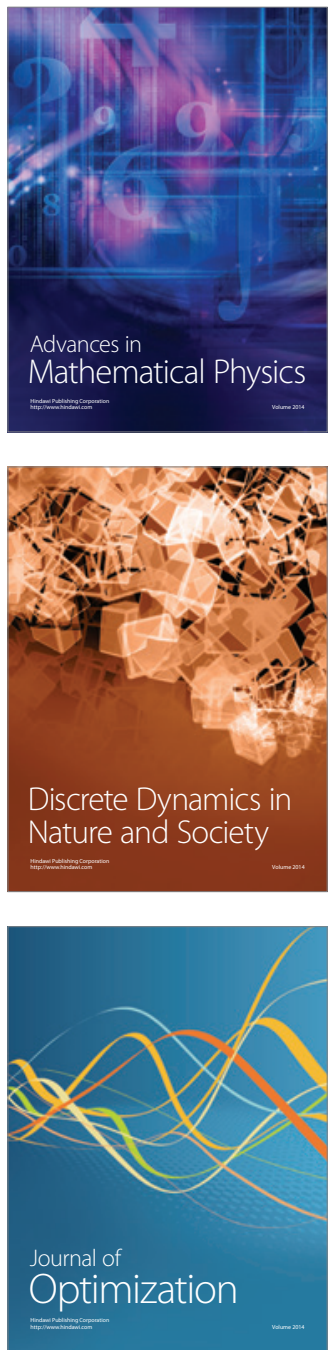